INPLASY

PROTOCOL

To cite: Xu et al. Extracorporeal shock wave in the treatment of Femur Head Necrosis: A metaanalysis of randomized controlled trials. Inplasy protocol 2021120003. doi: 10.37766/inplasy2021.12.0003

Received: 01 December 2021

Published: 01 December 2021

Corresponding author: Wang Lihe

wanglihe0222@sina.com

Author Affiliation: Department of Orthopedics and Traumatology, the First Affiliated Hospital of Henan University of Traditional Chinese Medicine.

Support: 2017ZY2044; $2019 Z$ YBJ06.

Review Stage at time of this submission: Preliminary searches.

Conflicts of interest: None declared.

\section{Extracorporeal shock wave in the treatment of Femur Head Necrosis: A meta-analysis of randomized controlled trials}

Xu, WB1; Wang, LH2; Wang, YD³; Gao, RY4; Jin, Z5; Liu, GQ66.

Review question / Objective: To evaluate the efficacy of extracorporeal shock wave in the treatment of patients with femoral head necrosis.

Condition being studied: Femoral head necrosis is an orthopedic disease with a high disability rate.It is estimated that there are approximately $\mathbf{8 . 1 2}$ million non-traumatic femoral head necrosis patients over the age of 15 in China, and the number of male patients is approximately twice that of female patients. The extremely high prevalence rate places a serious economic burden on the families of patients as well as on society. As a non-invasive therapy, extracorporeal shock wave therapy has the characteristics of low damage to tissues, low treatment risk, short cycle time and low complications.Related studies suggest that extracorporeal shock wave therapy can have a positive effect on the treatment of femoral head necrosis, but there is a lack of high-quality randomized controlled clinical trials. Therefore, the purpose of this study was to evaluate the efficacy of extracorporeal shock wave in the treatment of patients with femoral head necrosis.

INPLASY registration number: This protocol was registered with the International Platform of Registered Systematic Review and Meta-Analysis Protocols (INPLASY) on 01 December 2021 and was last updated on 01 December 2021 (registration number INPLASY2021120003).

\section{INTRODUCTION}

Review question / Objective: To evaluate the efficacy of extracorporeal shock wave in the treatment of patients with femoral head necrosis
Condition being studied: Femoral head necrosis is an orthopedic disease with a high disability rate.It is estimated that there are approximately 8.12 million nontraumatic femoral head necrosis patients over the age of 15 in China, and the number of male patients is approximately twice that 
of female patients. The extremely high prevalence rate places a serious economic burden on the families of patients as well as on society. As a non-invasive therapy, extracorporeal shock wave therapy has the characteristics of low damage to tissues, low treatment risk, short cycle time and low complications. Related studies suggest that extracorporeal shock wave therapy can have a positive effect on the treatment of femoral head necrosis, but there is a lack of high-quality randomized controlled clinical trials. Therefore, the purpose of this study was to evaluate the efficacy of extracorporeal shock wave in the treatment of patients with femoral head necrosis.

\section{METHODS}

Search strategy: \#1. search ( ( "Extracorporeal Shockwave Therapy"[Mesh]) OR \#2.search (l(()(()(()ESWT[Title/Abstract]) OR (Extracorporeal Shockwave Therapies[Title/Abstract])) OR (Shockwave Therapies, Extracorporeal[Title/Abstract])) OR (Shockwave Therapy, Extracorporeal [Title/Abstract])) OR (Therapy, Extracorporeal Shockwave[Title/Abstract])) OR (Shock Wave Therapy[Title/Abstract])) ) OR (Shock Wave Therapies[Title/Abstract])) OR (Shock Wave treatment[Title/Abstract])) OR (Therapy, Shock Wave[Title/Abstract])) OR (Extracorporeal Shock Wave Therapy[Title/Abstract]))) AND \#3.search(("Femur Head Necrosis"[Mesh])

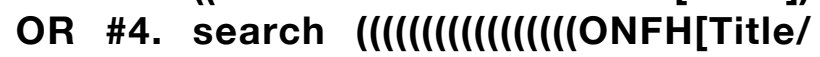
Abstract]) OR (Femur Head Necroses[Title/ Abstract])) OR (Head Necrosis, Femur[Title/ Abstract])) OR (Necrosis, Femur Head[Title/ Abstract])) OR (Aseptic Necrosis of Femur Head[Title/Abstract])) OR (Necrosis, Aseptic, of Femur Head[Title/Abstract])) OR (Necrosis, Avascular, of Femur Head[Title/ Abstract])) OR (Ischemic Necrosis Of Femoral Head[Title/Abstract])) OR (Femoral Head, Avascular Necrosis Of[Title/ Abstract])) OR (Avascular Necrosis Of Femoral Head, Primary[Title/Abstract])) OR (Avascular Necrosis of Femur Head[Title/ Abstract])) OR (femoral head osteonecrosis[Title/Abstract])) OR (femur head osteonecrosis[Title/Abstract])) OR (juvenile femur head necrosis[Title/
Abstract])) OR (necrosis, femur head[Title/ Abstract])) OR (osteonecrosis of the femoral head[Title/Abstract])) OR (osteonecrosis of the femur head[Title/ Abstract]))) \#5. search \#1 or \#2;\#6.search \#3 or \#4;\#7. search \#5 and \#6.

Participant or population: Patients with clinically diagnosed of femoral head necrosis will be fully considered for inclusion.

Intervention: In the experimental group, extracorporeal shock wave alone or extracorporeal shock wave combined with conventional interference measures (need to be the same as control group).

Comparator: In the control group, conventional interference measures, including drug treatment, Chinese medicine treatment, surgical treatment

Study designs to be included: Only randomized controlled trials(RCTs) will be included

Eligibility criteria: Inclusion Criteria:

(1)Research type: RCT(2)Participants: Patients with clinically diagnosed of femoral head necrosis(3)Outcome indicators :include at least one of the following: clinical efficiency,Harris hip score and The visual analogue score;Exclusion Criteria:(1)lt is not consistent with the direction of the subject of this study.(2)Duplicate published literature.(3)Literature with unknown data and unable to contact the authors for full details.(4)Literature on animal experiments, pediatric femoral head necrosis, conference summary literature, reviews, and systematic evaluations.(5)Not meeting the outcome indicators.

Information sources: The dadabases, including China National Knowledge Infrastructure (CNKI), Wangfang database, VIP database, Chinese Biomedical Literature Database (CBM), Pubmed, Web of Science, Cochrane Library and Embace will be searched to collect randomized controlled trails on the efficacy of 
extracorporeal shock wave in the treatment of femoral head necrosis from inception to September 30th, 2021, with no language and publication date restrictions We will also manually search other literatures to ensure that any potential eligible RCTs will not be missed.

Main outcome(s): Main outcomes include Clinical efficiency ,Harris hip score and The visual analogue score.

Data management: Two investigators independently will screen the literature and extract data strictly according to the inclusion and exclusion criteria. In case of inconsistent results, the controversial literature is first discussed and negotiated, and if it still can not be resolved, a third researcher is invited to join and discuss. A form for data extraction is pre-designed, which include the first author of the literature, year of publication, Experimental method, sample status, interventions, and outcome indicators.

Quality assessment / Risk of bias analysis: Two reviewers will independently assess the quality of the included literature according to the the Cochrane Risk of Bias Tool for RCTs, which is based on the following indicators: random sequence generation, allocation concealment, blinding of participants and personnel, blinding of outcome assessment, incomplete outcome data, selective reporting and other bias.

Strategy of data synthesis: The statistical analysis will be performed using RevMan v5.3 software which is provided by the Cochrane Collaboration Network. For dichotomous variables, we used relative risk(RR) with $95 \% \mathrm{Cl}$ as the effect measure. For continuous variables, we used mean difference(MD)or standardized mean difference(SMD)with $95 \% \mathrm{Cl}$ as the effect measure. Meta-analysis will be performed by a fixed-effects model when the heterogeneity is $\operatorname{low}(P>0.1,12<50 \%)$. If heterogeneity was high ( $P \leq 0.1,12 \geq 50 \%)$, the Meta-analysis will be performed using a random-effects model after excluding significant clinical heterogeneity.

Subgroup analysis: We will perform subgroup analysis to identify any possible sources of substantial heterogeneity based on different treatments, controls and other factors.

Sensitivity analysis: After deleting any one of them, the combined results of the remaining papers were not significantly different from those without deletion, which means that the sensitivity analysis was passed.

Country(ies) involved: China.

Keywords: Femoral head necrosis; extracorporeal shock wave therapy; metaanalysis, randomized controlled trials.

Contributions of each author:

Author 1 - Xu Wenbo.

Email: 15838234585@163.com

Author 2 - Wang Lihe.

Email: wanglihe0222@sina.com

Author 3 - Wang Yudong.

Author 4 - Gao Ruiyong.

Author 5 - Jin Zhu.

Author 6 - Liu Gaoqing. 